\title{
Reciclado de plásticos de tapas de botella y defensas automotrices reforzadas con fibra de vidrio para fabricar alcantarillas domésticas
}

\author{
Recycling plastics bottle caps and fiberglass-reinforced car bumpers plastics \\ for domestic sewers production
}

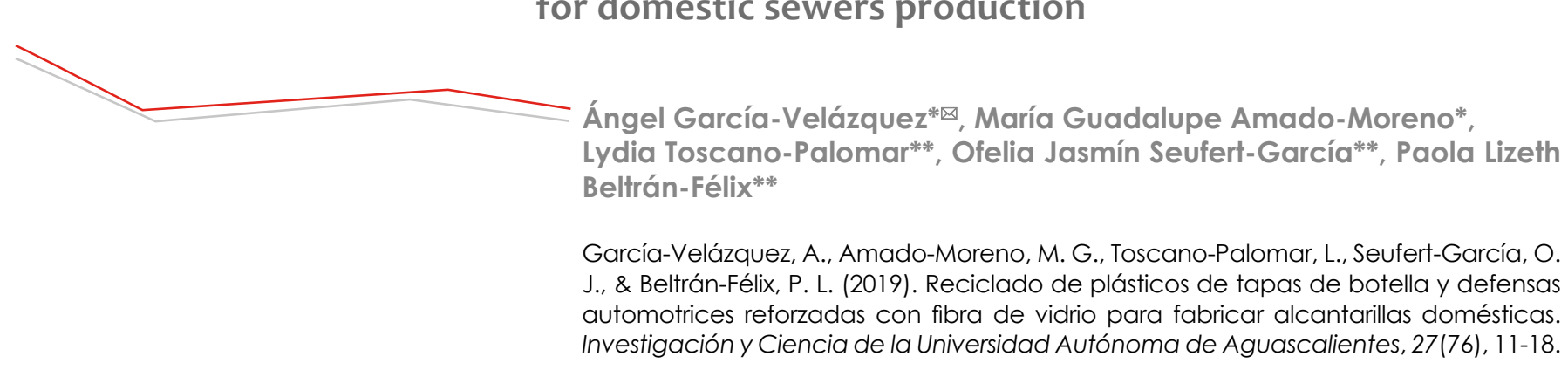

\section{RESUMEN}

El objetivo de esta investigación fue reciclar los plásticos de tapas de botella y defensas automotrices con un refuerzo de fibra de vidrio para fabricar alcantarillas domésticas. Se realizó un arreglo experimental considerando las tapas como resina principal, variando la concentración de fibra de vidrio y de plástico de defensa automotriz. Los resultados de las pruebas mecánicas, de absorción de agua, de consistencia y procesabilidad de la mezcla, determinaron que la formulación óptima para fabricar la alcantarilla fue $40 \%$ de plástico de defensas automotrices, $40 \%$ de fibra de vidrio y $1.5 \%$ de agente de acoplamiento. El proceso de elaboración, en comparación con los existentes en el mercado, es más económico y evita el consumo

Palabras clave: reciclado; tapas plásticas de botella; plástico de defensas automotrices; reciclado de polipropileno; materiales compuestos poliméricos.

Keywords: recycling; plastic bottle caps; car bumper plastic; polypropylene recycling; polymeric composite materials.

Recibido: 12 de diciembre de 2017, aceptado: 19 de septiembre de 2018

* Departamento de Ciencias Básicas, Instituto Tecnológico de Mexicali, Tecnológico Nacional de México. Avenida Tecnológico s/n, Colonia Elías Calles, C. P. 21376, Mexicali, Baja California, México. Correo electrónico: angel.g20@hotmail.com; lupitaamado@yahoo.com.mx. ORCID: orcid.org/0000-0002-8458-7755; orcid.org/0000-0001-6363-5888

** Departamento de Ingeniería Química y Bioquímica, Instituto Tecnológico de Mexicali, Tecnológico Nacional de México. Avenida Tecnológico s/n, Colonia Elías Calles, C. P. 21376, Mexicali, Baja California, México. Correo electrónico: Iydiatos@hotmail.com; jasminseufer@@hotmail.com; paolabelf@hotmail.com. ORCID: orcid.org/0000-0002-2472-4826; orcid. org/0000-0002-8222-0319; orcid.org/0000-0002-7257-8325

$凶$ Autor para correspondencia de combustibles fósiles. Es un producto que puede sustituir a las alcantarillas domésticas elaboradas con metales fundidos, es más ligero, fácil de moldear, resistente a la oxidación y duradero.

\section{ABSTRAC1}

The purpose of this research was to recycle plastic bottle caps and fiberglass-reinforced car bumpers for domestic sewer production. An experimental arrangement was made considering the bottle caps as the main resin with different concentrations of both fiberglass and car bumper plastic. The use of mechanic tests, water absorption, consistency and processability tests of mixture determined that the optimal formula to sewers production is composed of: $40 \%$ of car bumper plastic, $40 \%$ of fiberglass and $1.5 \%$ of coupling agent. While this procedure will not require fossil fuel consumption; it will also be found more economical than other procedures in the market. Its lightness; moldability, oxidative resistance and durability, would enable this product to substitute current domestic sewers; which are being produced with melted metals.

\section{INTRODUCCIÓN}

Reciclar plásticos es una opción para que un material de desecho se convierta en materia prima o en un nuevo producto. Hoy día muchos de los artículos de uso cotidiano se distribuyen en botellas con tapas de plástico, una vez desechadas se acumulan en calles y rellenos sanitarios; existe contaminación debida a plásticos de defensas automotrices, que al dañarse son difíciles de reparar y resulta más económica su 
issn 1665-4412, e-issn 2521-9758

García-Velázquez, A., Amado-Moreno, M. G., Toscano-Palomar, L., Seufert-

García, O. J., \& Beltrán-Félix, P. L sustitución, pero al no tener ninguna aplicación se acumulan en talleres mecánicos, desmanteladoras y calles.

En 2012 se generaron en México 37.5 millones de toneladas de residuos sólidos urbanos (a partir de aquí RSU). Baja California fue el octavo estado con mayor generación de RSU en el país, con 1,425.33 miles de $\dagger$ de residuos mixtos. Aproximadamente $10.9 \%$ de la basura en el país es plástico. La generación de RSU en 2015 aumentó, alcanzado 53.1 MMt, en donde también incrementó la cantidad de desechos plásticos (SEMARNAT, 2016).

Los residuos plásticos se degradan lentamente, lo que genera contaminación de suelos, ríos y mares, lo que constituye un problema para el país en materia ambiental y demanda a la sociedad la necesidad de reciclar. La contaminación generada por la disposición inadecuada de dichos materiales de desecho hizo posible desarrollar la investigación en el Instituto Tecnológico de Mexicali (ITM), en Baja California, México. Se utilizó el polipropileno (a partir de aquí PP) de tapas de botella y el plástico de defensas automotrices, ya que contiene aditivos especiales que favorecen las propiedades del material durante el proceso de reciclado (Pukánszky, 1999). Durante dicho proceso el plástico experimenta un decaimiento en sus propiedades mecánicas ocasionado por la modificación de su estructura molecular, en comparación con el material virgen. Para subsanar estas pérdidas se agregan materiales de refuerzo y aditivos, los cuales mejoran las propiedades del material reciclado (Callister \& Rethwisch, 2016; Hull, 2003). En los últimos años para mejorar el desempeño físico de los polímeros reforzados con fibras sintéticas se han utilizado diferentes agentes de acoplamiento, los cuales crean enlaces químicos entre el polímero y la fibra de refuerzo, lo que incrementa las propiedades del material compuesto (Sanjuan-Raygoza \& JassoGastinel, 2009).

Un material compuesto presenta dos elementos principales: fibra y matriz. La combinación adecuada de estos componentes origina materiales con mejores propiedades que las partes que los componen por separado. Los aditivos proporcionan a los materiales compuestos características peculiares para cada tipo de proceso y aplicación.

La fibra de vidrio es el refuerzo más utilizado debido a su bajo costo y propiedades, como alta resistencia a la tensión, a la intemperie, excelente estabilidad dimensional y baja conductividad térmica, además de ser incombustible y biológicamente inerte; por lo que cuando se une con un polímero sus propiedades se ven mejoradas (Miravete et al., 2007).

Los agentes de acoplamiento mejoran la adhesión entre el plástico y la fibra, reducen la absorción de agua e incrementan la resistencia mecánica. Además, ayudan a la dispersión de la fibra en la matriz, lo que puede permitir un incremento del porcentaje de fibra dentro del compuesto. Este tipo de aditivos se utiliza en concentraciones de 1 a 2\% (Flórez Sastre, 2009). El anhídrido maleico es uno de los mejores agentes de acoplamiento en las industrias del plástico y de los polímeros (Bustamante Rodríguez, Cañada Jaime, Madueña Mendoza, \& Medrano Urbano, 2001; Gallego, López, \& Gartner, 2006). Bouza Padín (2008) ha encontrado que para elaborar un material compuesto, la concentración de fibra de refuerzo varía de 20 a $40 \%$ y la del agente de acoplamiento de 0.5 a $5 \%$.

Existen investigaciones que abordan el reciclado del PP con diversas fibras, agentes de acoplamiento y plásticos. En Cuba se realizó reciclado de PP con bagazo cubano, una fibra vegetal, donde los mejores resultados obtenidos por Brown Gómez et al. (2007, p. 298) fueron "un $20 \%$ de fibra molida incorporada al material reforzado resulta el mejor valor para el porcentaje de cristalinidad y un aumento en la carga, dificulta la cristalización del material reforzado formulado", mencionan que se utilizó $5 \%$ de agente de acoplamiento para realizar la mezcla polimérica.

En Colombia, Córdoba Barahona, Mera, Martínez y Rodríguez (2010, p. 426) reciclaron PP con polietileno de alta densidad (a partir de aquí PEAD) y fibra vegetal mediante una concentración de $3 \%$ de fibra para todas las pruebas, encontraron como resultado que "todas las pruebas de compresión, flexión y tensión, muestran que la fibra no aumenta la resistencia, pero sí incrementa su ductilidad y esto puede ser aprovechado como ventaja para materiales que requieran ser livianos y muy deformables", se obtuvo que la más conveniente contenía $70 \%$ de PP y $30 \%$ de PEAD.

Rivai, Gupta, Islam y Beg (2014) reportan que en Malaysia se realizó reciclado de PP y como 
fibras de refuerzo utilizaron cáscaras de fruta de palma y fibra de vidrio, la mezcla polimérica fue con $40 \%$ de fibra, $60 \%$ de PP reciclado y $5 \%$ de anhídrido maleico Polybond 3200 como agente de acoplamiento. En México también se han realizado investigaciones sobre el reciclado del PP. Sanjuan-Raygoza y Jasso-Gastinel (2009) evaluaron la fibra de agave como refuerzo en un material compuesto con PP virgen y reciclado, en algunas formulaciones utilizaron agente de acoplamiento, encontraron que los mejores resultados en las pruebas mecánicas se obtuvieron cuando se utilizó agente de acoplamiento.

Lázaro León, Gonzáles Mora, Cárdenas Oscanoa y Gago Campusano (2016) evaluaron propiedades físicas de un material compuesto elaborado con PP reciclado y fibras de bambú, los mejores resultados se dieron con $30 \%$ de fibra de bambú, $70 \%$ de PP reciclado y anhidrído maleico de 0 a 2\%. Las investigaciones mencionadas motivaron la realización del presente trabajo, el cual consistió en el reciclaje de tapas plásticas de botella y defensas automotrices con refuerzo de fibra de vidrio y agente de acoplamiento para fabricar alcantarillas domésticas.

\section{MATERIALES Y MÉTODOS}

El material compuesto para elaborar las alcantarillas domésticas fue el PP como matriz y la fibra de vidrio como refuerzo. Debido a que dichos materiales son incompatibles químicamente (Miravete et al., 2007) se incorporó a la formulación anhídrido maleico como agente de acoplamiento y para mejorar la procesabilidad se añadió el plástico de defensas

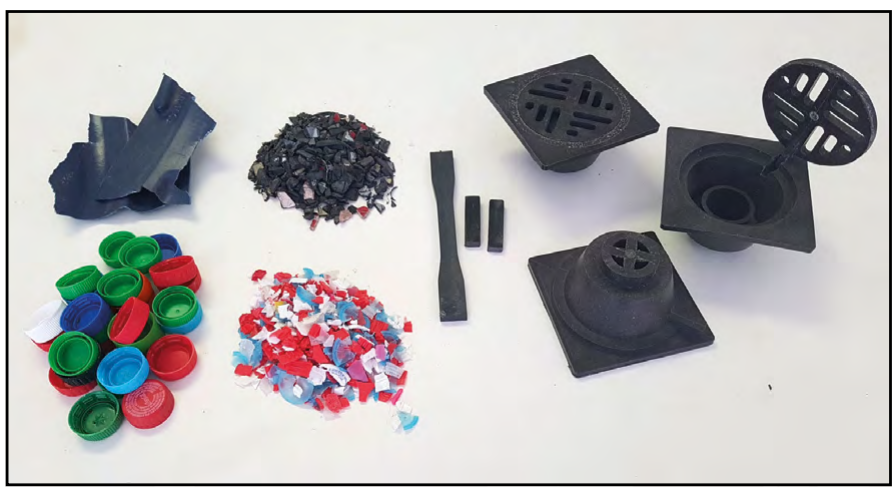

Figura 1. Tapas plásticas de botellas y defensas automotrices para fabricar alcantarillas domésticas.

Fotografía tomada por el equipo de investigación. automotrices, el cual contiene aditivos de ayuda de proceso que se mantienen en las defensas desde su fabricación (Pukánszky, 1999).

El proyecto se realizó en cinco etapas:

\section{1) Recolección y trituración de plástico de tapas de botellas y defensas automotrices}

Mediante una campaña de acopio las tapas plásticas de botella se recolectaron en el ITM, a su vez, las defensas automotrices se obtuvieron de talleres mecánicos y desmanteladoras en la Ciudad de Mexicali, Baja California, México. Estos materiales se trituraron por separado en un molino de dos hojas American Grinder de $5 \mathrm{HP}$, hasta un tamaño de partícula entre 2 y $7 \mathrm{~mm}$.

\section{2) Arreglo experimental}

Las variables en las formulaciones realizadas fueron la concentración de la fibra de vidrio y de plástico de defensa automotriz, el agente de acoplamiento se mantuvo fijo al $1.5 \%$ con respecto a la cantidad de polipropileno de tapas de botella que fue la resina principal, en la tabla 1 se observa el arreglo.

Tabla 1

Arreglo experimental para las formulaciones

\begin{tabular}{llllll}
\hline $\begin{array}{l}\text { \% de plástico } \\
\text { de defensa } \\
\text { automotriz }\end{array}$ & 0 & 30 & 40 & 50 \\
\hline 0 & $X$ & $X$ & & \\
20 & & & $X$ & & $X$ \\
30 & $X$ & $X$ & $X$ & \\
40 & & & & $X$ & \\
\hline
\end{tabular}

Nota: Elaboración propia.

Nomenclatura utilizada para las formulaciones:

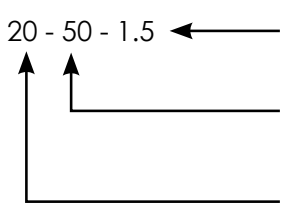
Porcentaje del agente de acoplamiento
respecto al total de polipropileno de tapas
Porcentaje de fibra de vidrio respecto al
total de polipropileno de tapas
Porcentaje de plástico de defensa respecto
al total de polipropileno de tapas

3) Elaboración del material compuesto según las formulaciones

Con el material triturado se prepararon las ocho formulaciones señaladas en la tabla 1. Para el 
issn 1665-4412, e-issn 2521-9758

García-Velázquez, A., Amado-Moreno, M. G., Toscano-Palomar, L., Seufert-

García, O. J., \& Beltrán-Félix, P. L mezclado se utilizó un extrusor monohusillo (ver figura 2) con una relación $L / D=20 / 1$ accionado por una unidad de potencia hidráulica y operando a una velocidad de $40 \mathrm{rpm}$ en el husillo.

Se generó un extruido, con una sección transversal de $3 \mathrm{~mm}$ de diámetro, el cual fue enfriado en agua a $3^{\circ} \mathrm{C}$. Una vez a temperatura ambiente fue seccionado en una peletizadora. En la figura 3 se presenta el material compuesto extruido y peletizado.

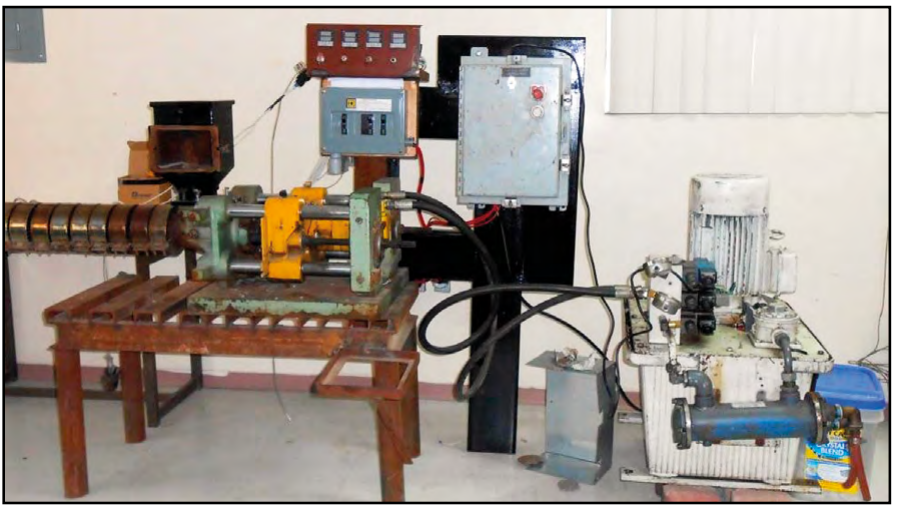

Figura 2. Extrusor monohusillo.

Fotografía tomada por el equipo de investigación.

4) Elaboración de probetas para pruebas del material compuesto

Las dimensiones de las probetas y el procedimiento de prueba se realizaron según las normas de la American Society for Testing and Materials (ASTM) mostradas en la tabla 2, así como el equipo utilizado. Se elaboraron las probetas para las ocho formulaciones obtenidas. La técnica utilizada fue moldeo por inyección.

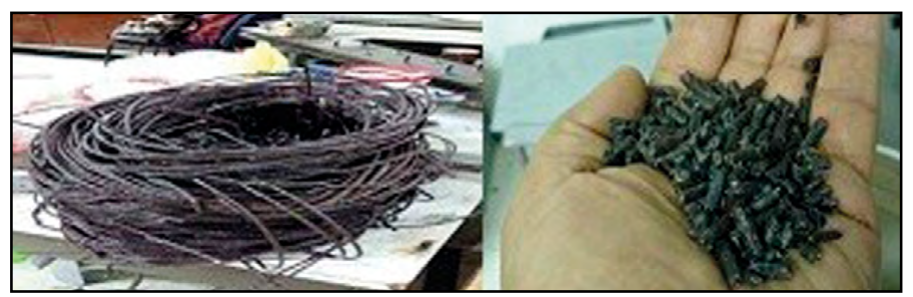

Figura 3. Material compuesto extruido y peletizado.

Fotografía tomada por el equipo de investigación.

Prueba de flexión. Con esta prueba se midió la capacidad que tiene el material de doblarse sin romperse, debido a la aplicación de una carga. Se elaboraron 10 probetas para cada una de las formulaciones.

Prueba de tensión. Se determinó el máximo esfuerzo que el material puede resistir antes de su rotura cuando es tensionada por ambos extremos, el módulo elástico y el porcentaje de elongación. En la figura 4 se muestra el molde maquinado en acero inoxidable 304 y la probeta obtenida para la prueba de tensión. Se elaboraron 10 probetas para cada una de las formulaciones.

Prueba de resistencia al impacto. Para esta prueba se realizaron cinco probetas para cada formulación, algunas se observan en la figura 5. Posteriormente se midió la energía absorbida en la rotura de las muestras.

Prueba de absorción de humedad. Las probetas fueron secadas durante $24 \mathrm{~h}$ a $110^{\circ} \mathrm{C}$, pesadas e introducidas en agua destilada durante $24 \mathrm{~h}$, al cumplirse el tiempo se les retiró el agua de la superficie y se pesaron de nuevo. El contenido de

Tabla 2

Normatividad y equipo para las pruebas mecánicas, absorción de humedad e índice de fluidez

\begin{tabular}{|c|c|c|}
\hline Prueba & Norma & Equipo \\
\hline Flexión & $\begin{array}{l}\text { A } S \text { T M } \\
\text { D-790-02 }\end{array}$ & $\begin{array}{l}\text { Máquina universal de ensayos marca Physical } \\
\text { Test Solutions (PTS) }\end{array}$ \\
\hline Tensión & $\begin{array}{l}\text { A } S \text { T M } \\
\text { D638 }\end{array}$ & \\
\hline $\begin{array}{l}\text { Resistencia al } \\
\text { impacto }\end{array}$ & $\begin{array}{l}\text { A S T } M \\
\text { D256-93 }\end{array}$ & $\begin{array}{l}\text { Máquina de impacto Monsanto Tensómetro } \\
\text { de doble brazo modalidad Charpy }\end{array}$ \\
\hline $\begin{array}{l}\text { Absorción de } \\
\text { humedad }\end{array}$ & $\begin{array}{l}\text { A } S \text { T } M \\
\text { D-570-98 }\end{array}$ & Método de diferencia de pesos \\
\hline Índice de fluidez & $\begin{array}{l}\text { A } S \text { T } M \\
\text { D-1238 }\end{array}$ & $\begin{array}{l}\text { Medidor de índice de fluidez. Modelo MFI1312 } \\
\text { marca Physical Test Solutions (PTS) }\end{array}$ \\
\hline
\end{tabular}

Nota: Elaboración propia. 


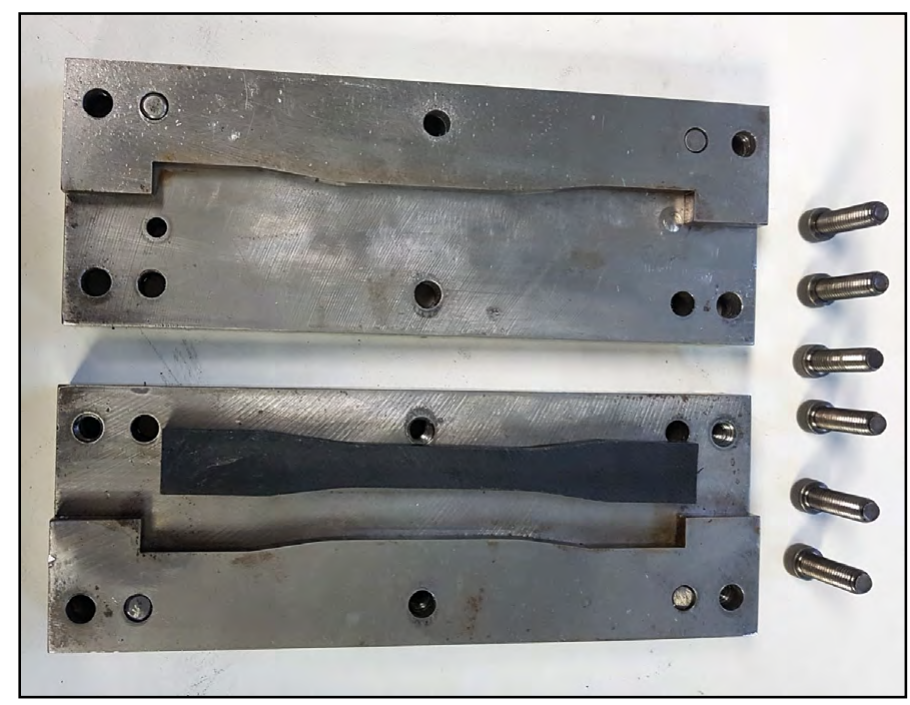

Figura 4. Molde y probeta para la prueba de tensión. Fotografía tomada por el equipo de investigación.

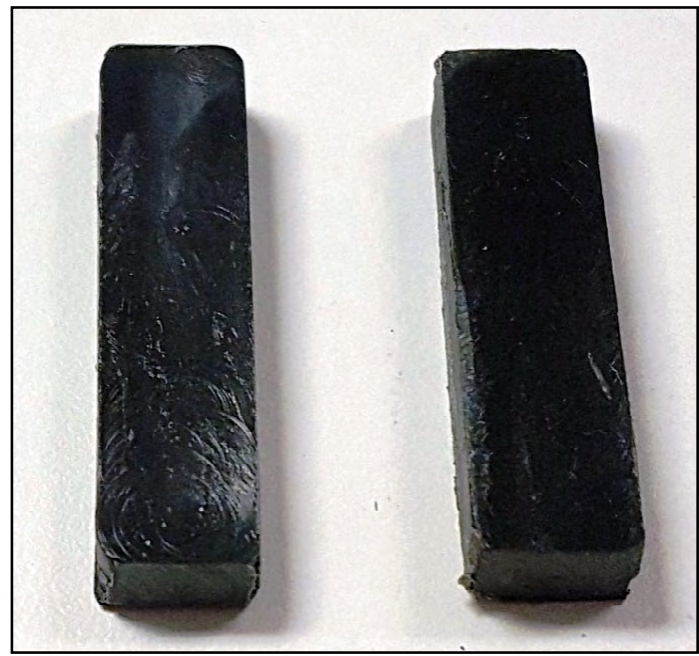

Figura 5. Probetas para la prueba de impacto. Fotografía tomada por el equipo de investigación.

humedad se determinó por diferencia de pesos para cada una de las ocho formulaciones.

Índice de fluidez. Es una prueba reológica que se realizó para las ocho formulaciones. Se define como la cantidad de plástico que fluye a través de un dado en 10 min, manteniendo constante presión y temperatura. Se relaciona con el peso molecular del plástico y su procesabilidad.

\section{5) Diseño y elaboración del molde para la fabricación de la alcantarilla doméstica}

Se diseñó un molde de dos piezas para el

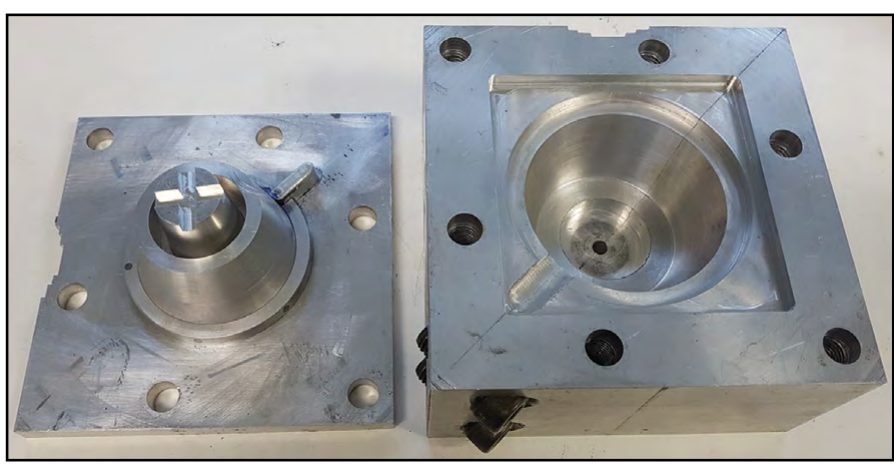

Figura 6. Molde de dos piezas para el cuerpo de la alcantarilla. Fotografía tomada por el equipo de investigación.

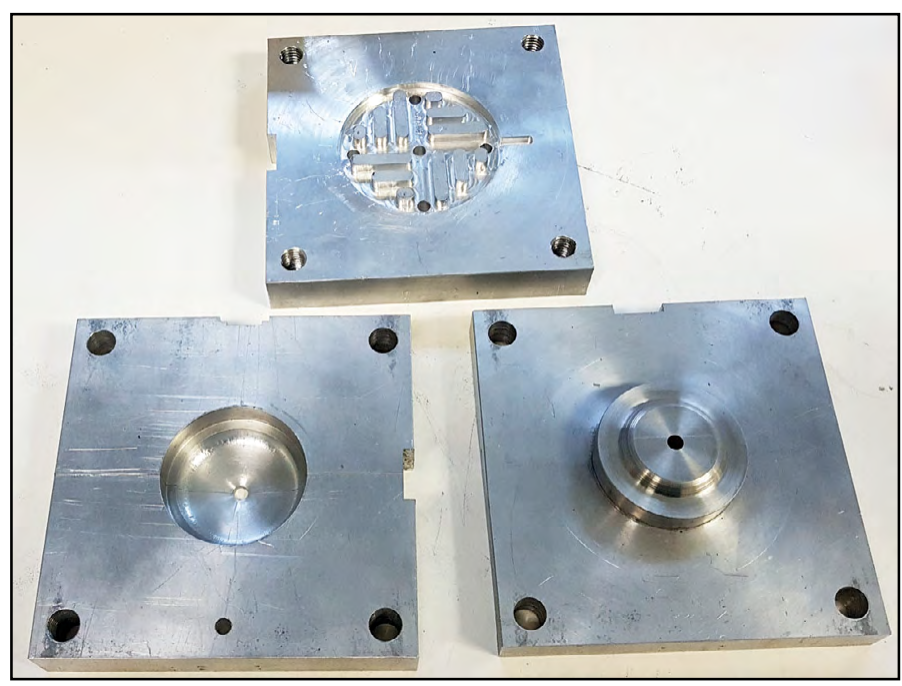

Figura 7. Molde de tres piezas para la rejilla de la alcantarilla. Fotografía tomada por el equipo de investigación.

cuerpo de la alcantarilla y otro de tres piezas para la inyección de la rejilla. En las figuras 6 y 7 se observan los dos moldes. Se elaboró con medidas comerciales: base de 4" $\times 4 "$ ", entrada para tubo de drenaje de 1.5" y trampa integrada para la retención de sólidos y olores.

\section{RESULTADOS}

Se presentan en la tabla 3 los resultados de la prueba de flexión, las formulaciones que presentaron mayor resistencia a la flexión fueron 20-50-1.5, 40-40-1.5 y $100 \%$ de tapas. También es posible visualizar que las dos formulaciones con mayor resistencia a la flexión son las que tienen mayor concentración de fibra de vidrio. 
issn 1665-4412, e-issn 2521-9758

García-Velázquez, A., Amado-Moreno,

M. G., Toscano-Palomar, L., Seufert-

García, O. J., \& Beltrán-Félix, P. L
Tabla 3

Resultados de la medición de la prueba flexión

\begin{tabular}{lcc}
\hline Formulación & $\begin{array}{c}\text { Resistencia a la flexión } \\
\text { (MPa) }\end{array}$ & $\begin{array}{c}\text { Desviación } \\
\text { estándar }\end{array}$ \\
\hline $20-50-1.5$ & 613.443 & 18.191 \\
$40-40-1.5$ & 578.914 & 35.364 \\
$100 \%$ tapas & 564.321 & 44.540 \\
$30-40-1.5$ & 559.221 & 23.497 \\
$0-30-1.5$ & 434.231 & 33.867 \\
$30-30-1.5$ & 412.174 & 45.601 \\
$20-30-1.5$ & 344.677 & 17.404 \\
$30-0-0$ & 256.736 & 25.161 \\
\hline
\end{tabular}

Nota: Elaboración propia.

En la tabla 4 se observó que las formulaciones 20-50-1.5, 40-40-1.5 y 30-40-1.5 presentaron el valor máximo del módulo elástico y mayor cantidad de fibra de vidrio. En cuanto al porcentaje de elongación no se observa mucha variación, a excepción de las formulaciones $30-0-0$ y $100 \%$ tapas, que presentan mayor porcentaje de elongación, el resto tiene un porcentaje de elongación entre 9 y $19 \%$. Las tres formulaciones con esfuerzo máximo a la rotura fueron $40-40-1.5,20-50-1.5$ y 30-40-1.5, las que tienen mayor concentración de fibra de vidrio.

Las formulaciones que mostraron mayor resistencia al impacto se observan en la tabla 5 y fueron 20-50-1.5, 40-40-1.5, 20-30-1.5, 30-40-1.5 y 3030-1.5.
Tabla 5

Resultados de la medición de la prueba de impacto

\begin{tabular}{lcc}
\hline Formulación & $\begin{array}{c}\text { Resistencia al } \\
\text { impacto Isod }\left(\mathrm{KJ} / \mathrm{m}^{2}\right)\end{array}$ & $\begin{array}{c}\text { Desviación } \\
\text { estándar }\end{array}$ \\
\hline $20-50-1.5$ & 44.344 & 2.713 \\
$40-40-1.5$ & 41.484 & 4.672 \\
$20-30-1.5$ & 40.850 & 3.806 \\
$30-40-1.5$ & 40.830 & 1.586 \\
$30-30-1.5$ & 40.070 & 3.664 \\
100\% tapas & 38.560 & 0.237 \\
$0-30-1.5$ & 35.400 & 3.754 \\
$30-0-0$ & 12.787 & 14.629 \\
\hline
\end{tabular}

Nota: Elaboración propia.

Se observa en la tabla 6 que el porcentaje de absorción de humedad en todas las formulaciones se mantuvo bajo y con una ligera variación.

Tabla 6

Resultados de la prueba de absorción de humedad

\begin{tabular}{lcc}
\hline Formulación & $\begin{array}{c}\text { \% de absorción de } \\
\text { humedad }\end{array}$ & $\begin{array}{c}\text { Desviación } \\
\text { estándar }\end{array}$ \\
\hline $20-50-1.5$ & 0.056 & 1.251 \\
$40-40-1.5$ & 0.042 & 6.343 \\
$30-40-1.5$ & 0.038 & 2.165 \\
$0-30-1.5$ & 0.035 & 2.851 \\
$30-0-0$ & 0.033 & 3.771 \\
$100 \%$ tapas & 0.032 & 4.024 \\
$20-30-1.5$ & 0.027 & 3.610 \\
$30-30-1.5$ & 0.024 & 2.186 \\
\hline
\end{tabular}

Nota: Elaboración propia.

Tabla 4

Resultados de la medición de la prueba de tensión

\begin{tabular}{lcccccc}
\hline Formulación & $\begin{array}{c}\text { Módulo } \\
\text { elástico (MPa) }\end{array}$ & $\begin{array}{c}\text { Desviación } \\
\text { estándar }\end{array}$ & $\begin{array}{c}\text { \% de } \\
\text { elongación }\end{array}$ & $\begin{array}{c}\text { Desviación } \\
\text { estándar }\end{array}$ & $\begin{array}{c}\text { Esfuerzo máximo } \\
\text { (MPa) }\end{array}$ & $\begin{array}{c}\text { Desviación } \\
\text { estándar }\end{array}$ \\
\hline $20-50-1.5$ & 3.292 & 0.251 & 12.038 & 1.074 & 38.928 & 0.482 \\
$40-40-1.5$ & 3.180 & 0.194 & 12.939 & 4.902 & 40.807 & 0.335 \\
$30-40-1.5$ & 2.920 & 0.124 & 13.053 & 7.152 & 37.921 & 1.256 \\
$30-30-1.5$ & 2.714 & 0.188 & 13.835 & 1.568 & 21.473 & 0.455 \\
$20-30-1.5$ & 2.662 & 0.230 & 15.200 & 2.255 & 21.380 & 0.633 \\
$30-0-0$ & 2.112 & 0.052 & 137.918 & 138.878 & 19.217 & 0.231 \\
$0-30-1.5$ & 2.100 & 0.231 & 9.840 & 1.687 & 15.554 & 0.255 \\
$100 \%$ tapas & 1.482 & 0.850 & 18.709 & 3.613 & 19.016 & 0.704 \\
\hline
\end{tabular}

Nota: Elaboración propia. 
En la tabla 7 se observa que las formulaciones con mayor índice de fluidez fueron 30-0-0, 20-30-1.5, 30-30-1.5, 30-40-1.5 y 40-40-1.5.

Tabla 7

Resultados de la medición de índice de fluidez

\begin{tabular}{lcc}
\hline Formulación & $\begin{array}{c}\text { Promedio del } \\
\text { indice de fluidez } \\
\text { (g/10min) }\end{array}$ & Desviación estándar \\
\hline $30-0-0$ & 39.817 & 7.493 \\
$20-30-1.5$ & 39.713 & 8.248 \\
$30-30-1.5$ & 32.049 & 4.599 \\
$30-40-1.5$ & 31.205 & 7.593 \\
$40-40-1.5$ & 26.278 & 11.603 \\
$0-30-1.5$ & 6.888 & 4.785 \\
$20-50-1.5$ & 6.812 & 5.222 \\
$100 \%$ tapas & 4.176 & 3.450 \\
\hline
\end{tabular}

Nota: Elaboración propia.

\section{DISCUSIÓN}

La formulación con mejores propiedades mecánicas fue 20-50-1.5, ya que contiene una concentración alta de fibra de vidrio; sin embargo, durante la extrusión se presentó separación de la fibra y el plástico por un débil anclaje. El índice de fluidez fue de los más bajos, lo cual puede asociarse con el alto porcentaje de fibra de vidrio dificultando la procesabilidad, por lo cual se descartó como formulación para el moldeo por inyección de las alcantarillas domésticas. La formulación 4040-1.5 fue la segunda con mejores propiedades mecánicas, contiene una concentración alta de fibra de vidrio, presenta consistencia homogénea e índice de fluidez alto, lo cual puede asociarse al alto porcentaje de defensas automotrices y a los aditivos que estas le confieren.

Las formulaciones 30-40-1.5, 30-30-1.5 y 2030-1.5 presentaron buen anclaje entre la fibra de vidrio y el plástico, así como un índice de fluidez mayor que las formulaciones 20-50-1.5 y 40-401.5. Las propiedades mecánicas son superiores en porcentaje de elongación a las de 20-50-1.5 y 4040-1.5, pero menores en módulo elástico, esfuerzo máximo a la rotura y resistencia al impacto. El porcentaje de absorción de humedad en todas las formulaciones se mantuvo bajo. Los resultados de la prueba de humedad validan que el polipropileno es un material idóneo para reciclar, ya que mantiene la propiedad de no ser higroscópico (Mink, 1990).
Finalmente se seleccionó como formulación óptima la 40-40-1.5, su buena procesabilidad al momento del moldeo por inyección permitió llenar las cavidades de los moldes sin dificultad y con el acabado adecuado, con ella se fabricaron las alcantarillas mostradas en la figura 8.

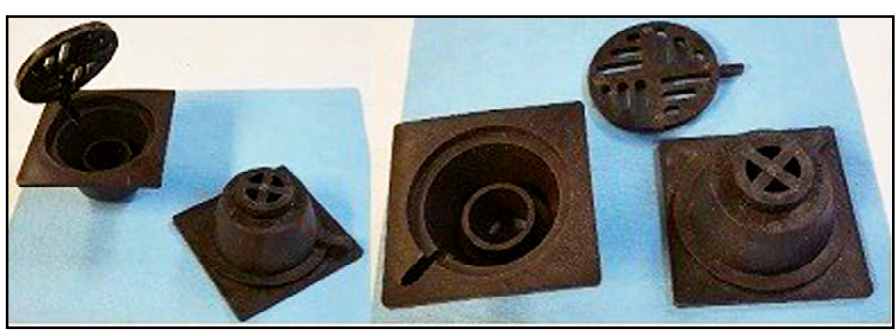

Figura 8. Alcantarilla doméstica elaborada con plástico reciclado. Fotografía tomada por el equipo de investigación.

CONCLUSIONES

En este proyecto se recicló polipropileno de tapas plásticas de botella y defensas automotrices con refuerzo de fibra de vidrio y agente de acoplamiento. La formulación óptima del material compuesto obtenido fue 40-40-1.5; es decir, aquella que contiene $40 \%$ de plástico de defensas de automóvil, $40 \%$ de fibra de vidrio y $1.5 \%$ de agente de acoplamiento, porcentajes referidos a la cantidad total de polipropileno de las tapas. Con esta formulación se fabricaron las tapas para desagüe domésticas.

Los criterios cuantitativos para la selección de la formulación óptima fueron la resistencia al impacto y a la flexión, el módulo elástico, el porcentaje de elongación, el esfuerzo máximo y el índice de fluidez; mientras que el criterio cualitativo fue la consistencia o anclaje entre la fibra de vidrio y el plástico.

La aportación principal de esta investigación fue la elaboración de alcantarillas domésticas con materiales plásticos de desecho, y se obtuvo una formulación óptima susceptible de ser utilizada para elaborar otros productos.

La alcantarilla producida con plásticos reciclados es un material más ligero, manejable, resistente a la oxidación y duradero con respecto a las tapas para desagüe tradicionales. Es un producto que puede sustituir a los sumideros domésticos elaborados con metales fundidos. El proceso de elaboración de la rejilla obtenida en comparación con las existentes en el mercado es más económico 
issn 1665-4412, e-issn 2521-9758

García-Velázquez, A., Amado-Moreno, M. G., Toscano-Palomar, L., SeufertGarcía, O. J., \& Beltrán-Félix, P. L y evita el consumo de combustibles fósiles, así como la disminución de los residuos plásticos en rellenos sanitarios.

Al reprocesar plásticos sus propiedades disminuyen, la fibra de vidrio permite recuperar propiedades mecánicas pero aumenta la viscosidad, por lo que se requieren ayudas de proceso (aditivos) que se encuentran en otro material plástico que también se encuentra contaminado el medio ambiente: las defensas automotrices, por lo que combinados son una alternativa para el reciclado.

El reciclaje con materiales plásticos representa un área de oportunidad para aplicaciones en el sector industrial. En la fabricación de tubería para drenaje, conectores, coplees, codos, tés, reducciones, cajas de contactos e interruptores, contenedores para pintura, aceites, adhesivos, impermeabilizantes entre otros.

\section{REFERENCIAS}

- Bouza Padín, R. (2008). Diseño y caracterización de nuevos materiales compuestos polipropileno y madera: Estudio del viniltrimetoxisilano como agente de acoplamiento (Tesis doctoral, Universidad de A Coruña, España). Recuperado de http://ruc.udc.es/dspace/bitstream/handle/2183/7356/ Bouza_Rebeca_TD_2008.pdf? sequence=1

- Brown Gómez, A., Matellanes, L., Álvarez Delgado, A., Momeñe, E., Txarroalde, J. R., Serantes, M., Leal, J. A., \& Renté, A. (2007). Disminución del empleo de materiales plásticos a partir de la revalorización del bagazo cubano. Revista Iberoamericana de Polímeros, 8(4), 287-299. Recuperado de https://dialnet.unirioja.es/servlet/articulo? codigo=2382107

- Bustamante Rodríguez, A., Cañada Jaime, H., Madueña Mendoza, F. J., \& Medrano Urbano, J. C. (2001). Diseño de una planta para la fabricación de anhídrido maléico vía n-butano (Proyecto final, Universidad Autónoma Metropolitana, México). Recuperado de https://www. academia.edu/7924893/J_DISE\%C3\%910_DE_UNA_PLANTA_PARA_LA_FABRICACI\%C3\%B3N_DE_ANH\%C3\%8DDRIDO_ MAL\%C3\%89ICO_VIA_N-BUTANO_--_AUTORES

- Callister, W. D., \& Rethwisch, D. G. (2016). Ciencia e ingeniería de materiales ( $2^{a}$. ed.). España: Reverté.

- Córdoba Barahona, C., Mera, J., Martínez, D., \& Rodríguez, J. (2010). Aprovechamiento de polipropileno y polietileno de alta densidad reciclados, reforzados con fibra vegetal, tetera (Stromanthe stromathoides). Revista Iberoamericana de Polímeros, 11 (7), 417-427. Recuperado de https://dialnet. unirioja.es/servlet/articulo? codigo $=3694217$

- Flórez Sastre, L. (2009). Desarrollo, tendencias y perspectiva del mercado de las maderas plásticas. Tecnología del Plástico, 1-3. Recuperado de http://www.plastico.com/temas/ Desarrollos,-tendencias-y-perspectiva-del-mercado-de-lasmaderas-plasticas +3072802

- Gallego, K., López, B. L., \& Gartner, C. (2006). Estudio de mezclas de polímeros reciclados para el mejoramiento de sus propiedades. Revista Facultad de Ingeniería de la Universidad de
Antioquia, 37, 59-70. Recuperado de http://www.redalyc.org/ pdf/430/43003707.pdf

- Hull, D. (2003). Materiales compuestos. España: Reverté.

- Lázaro León, K. C., Gonzáles Mora, H. E., Cárdenas Oscanoa, A. J., \& Gago Campusano, J. (2016). Evaluación de las propiedades físicas de material compuesto elaborado con bambú (Guadua angustifolia Kunth) y polipropileno. Revista Mexicana de Ciencias Forestales, 7(38), 79-94. Recuperado de http://www.scielo.org.mx/scielo.php?.pid=\$2007$11322016000600079 \&$ script=sci_arttext

- Mink, W. (1990). El plástico en la industria. Tratado práctico. Inyección de plásticos. México: G.Gili.

- Miravete, A., Larrodé, E., Castejón, L., Clemente, R., Jiménez, M. A., Lizaranzu, M., ... Peralta, J. L. (2007). Materiales Compuestos I. España: Reverté.

- Pukánszky, B. (1999). Particulate filled polypropylene composites. En J. Karger-Kocsis (Ed.), Polypropylene: An A-Z Reference (pp. 574-657). Budapest: Kluwer Publishers.

- Rivai, M., Gupta, A., Islam, M. R., \& Beg, M. D. H. (2014). Characterization of oil palm empty fruit bunch and glass fiber reinforced recycled polypropylene hybrid composites. Fibers and Polymers, 15(7), 1523-1530. doi: 10.1007/s12221-0141523-y

- Sanjuan-Raygoza, R. J., \& Jasso-Gastinel, C. F. (2009). Efecto de la fibra de agave de desecho en el reforzamiento de polipropileno virgen o reciclado. Revista Mexicana de Ingeniería Química, 8(3), 319-327. Recuperado de http:// wWw.scielo.org.mx/scielo.php?script=sci_arttext\&pid $=$ S1665-27382009000300011

- Secretaría de Medio Ambiente y Recursos Naturales. (2016). Informe de la situación del medio ambiente en México 2015. Compendio de estadísticas ambientales, indicadores clave, de desempeño ambiental y de crecimiento verde. Recuperado de http://apps1.semarnat.gob.mx/dgeia/informe15/ tema/pdf/Informe15_completo.pdf 